\title{
Sleeping Beauty jumps to new heights
}

\author{
Friedrich C. Luft
}

Published online: 14 May 2010

(C) Springer-Verlag 2010

Transposons are segments of DNA that can move around to different positions in the genome of a single cell [1]. In the process, they may cause mutations and thereby increase (or decrease) the amount of DNA in the genome. These mobile segments of DNA are sometimes called "jumping genes". There are two distinct types of transposons. Class I transposons (retrotransposons) first transcribe the DNA into RNA and then use reverse transcriptase to make a DNA copy of the RNA to insert in a new location. Class II transposons consist only of DNA that moves directly from place to place. Most Class II transposons move by a "cutand-paste" process (rather like your personal computer if you rely on the command- $X$ and command- $V$ function). The transposon is cut out of its location analogous to the command/control-X on a computer. The transposon is then inserted into a new location with the command/control- $\mathrm{V}$ maneuver. The ends of many transposons contain inverted repeats that are identical or nearly identical in sequence and read in opposite directions (Fig. 1); these inverted repeats are required for the transposition process. Some transposons require a specific sequence as their target site; others can insert anywhere in the genome. The DNA at the target site is cut in an offset manner similar to the "sticky ends" produced by restriction enzymes. After the transposon is inserted into the host DNA, the gaps are filled in by means of Watson-Crick base pairing. The base pairing creates identical direct repeats flanking each end of the transposon. A specific enzyme is required for transposons to work, namely a transposase, which is encoded within some, but not all, transposons. The transposase binds to the inverted

F. C. Luft $(\bowtie)$

Experimental and Clinical Research Center, Franz-Volhard Clinic,

Berlin, Germany

e-mail: luft@charite.de repeats at both ends of the transposon. The function and structure of some transposases (Fig. 2) have been worked out in detail $[2,3]$. Sometimes transposons lose their gene for transposase. However, as long as somewhere in the cell there is a transposon that can synthesize the enzyme, their inverted repeats are recognized by the available transposase and they also can be moved to a new location.

Barbara McClintock worked with maize and discovered transposons in the 1940s [4]. She observed that in developing somatic maize tissues, like corn kernels for example, a mutation can occur (for instance one that alters color) that can be passed on to all daughter cells. McClintock was a cytogeneticist who learned how to stain, and thereby identify, the 10 chromosomes of the maize plant. McClintock was able to observe the crossing-over effect between homologous chromosomes during meiosis. She also observed the separation of the centromeres and organization of the nucleolus. She was able to irradiate the plants, she described a massive increase in the mutation rate, and she recognized the importance of the chromosomal caps, the telomeres. Her most memorable observation was the identification of a spontaneous breakpoint on chromosome 9 of the maize plant. This breakpoint was associated with the color of various corn kernels of the plant. McClintock termed this breakpoint "dissociator" $(D s)$. After painstaking observations, she reached the conclusion that $D s$ was able to change its position on the chromosome and she named $D s$ a "jumping" gene. The world finally jumped when McClintock was awarded the Nobel Prize for physiology and Medicine in 1983.

Ivics et al., in the laboratory group of Perry Hackett, identified a practicable transposon system that could be used to insert genes in a therapeutic fashion [5]. Briefly, the group found a fish gene, dormant for probably 15 million years, which they turned into a vehicle for integrating 

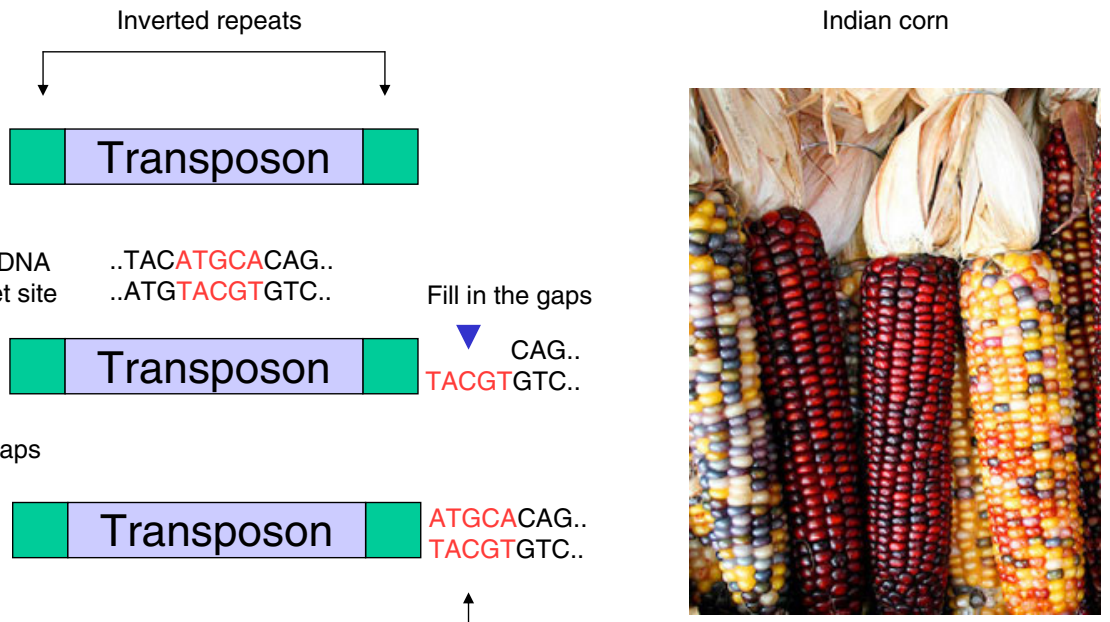

\section{Direct repeats}

Fig. 1 An inverted repeat is a sequence of nucleotides that is the reversed complement of another sequence further downstream. Both ends of the transposon that consists of inverted repeats, namely identical, or nearly identical, are required for the transposon to jump. Some transposons require a certain sequence as their target, while

foreign genes into chromosomes, potentially even human chromosomes. They named this transposon system "Sleeping Beauty". Since then, Sleeping Beauty has had little rest. The International Society for Molecular and Cell Biology and Biotechnology Protocols and Researches, named Sleeping Beauty the "Molecule of the Year 2009". The designation "Molecule of the Year" had erstwhile been conferred by the journal Science. Sleeping Beauty's DNA sequence had mutated in fish genomes to the point where the transposon

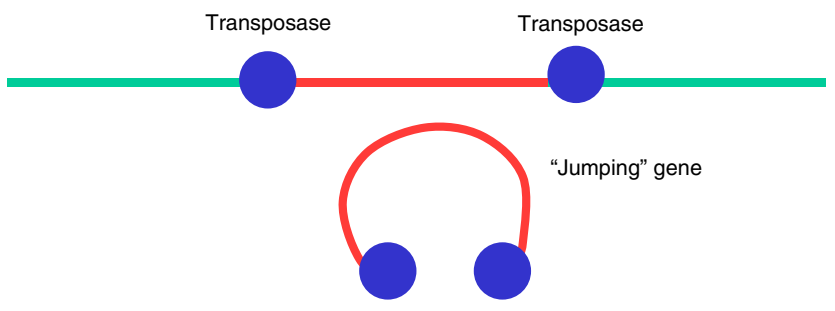

Target DNA
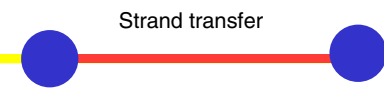

Target DNA

Fig. 2 The mechanism of "cut-and-paste" transposition is shown. First, the transposase (blue spheres) binds to specific sites at the ends of the transposon DNA (red). Then, transposon DNA looping results in formation of a complex that brings the two ends of the transposable element close together. Once the complex has been formed, the transposase cuts the transposon DNA away from the flanking "donor" DNA (green). After cleavage, transposase/DNA complex can move about freely until it encounters and binds to the "target" DNA (yellow) others can land anywhere in the genome. However, the transposon requires a transposase to function. Shown are the colorful kernels of Indian corn. Barbara McClintock explained the differences in kernel color on the basis of a somatic "jumping gene", namely a transposon. For this idea, she was awarded the Nobel Prize

no longer jumped, but rather slumbered as inactive junk DNA'. The extinct Sleeping Beauty jumping functions had to first be resurrected. Ivics et al. [6] used molecular phylogenetic data to construct a synthetic Sleeping Beauty. This utilitarian Sleeping Beauty is likely equivalent to the ancient element that dispersed in fish genomes probably by horizontal transmission between species. The investigators engineered a consensus sequence of a transposase gene by eliminating the inactivating mutations. They found that the Sleeping Beauty transposase binds to the inverted repeats of fish transposons, and that it mediates precise, "cut-and-paste" transposition in fish, mouse, and human cells. Thereby, Ivics et al. [5] produced the synthetic Sleeping Beauty, an active DNA-transposon system from vertebrates, to perform insertional mutagenesis, to conduct genetic transformation, and possibly to cure genetic diseases in humans.

How could these processes work and do we have evidence that they do? Many agents have been used to produce mutagenesis, including radiation as employed by Barbara McClintock, chemicals such as the ethyl-nitrosourea currently en vogue, or viruses that are used to disrupt genes randomly with the hope of identifying gene functions and the associated diseases. However, finding where any given mutation occurred in the genome is difficult. Transposable elements could help because their sequence is known, so when they mutate genes, they could provide a 'tag' that pinpoints their location in the genome. Tagging genes by insertion is not a new idea, and geneticists working on many organisms already use transposable elements. Mammals (mice) have not been of utility because known transposons are not doing much jumping in the mouse. Collier et al. [6] produced transgenic mice harboring the Sleeping Beauty transposon. The mouse 
line was prone to the development of cancers. These mice were mated with another mouse strain transgenic for the respective transposase. The excised transposon could then reintegrate elsewhere in the genome, sometimes close to or within a cancer-related gene. If the transposon is inserted into a gene, it would truncate the encoded protein, usually destroying its function. This action would identify genes that help to protect against cancer, like tumor-suppressor genes for example. Inserting near a gene can also cause an increase in gene product. This effect would identify cancer-promoting genes (oncogenes). Collier et al. [6] reported the ability of the Sleeping Beauty transposon to act as a somatic insertional mutagen to identify genes involved in solid tumor formation.

Could Sleeping Beauty have therapeutic utility in terms of introducing helpful transgenes [7]? Belcher et al. [8] tested this hypothesis as reported in this issue of J Mol Med. Their goal was to express the heme oxygenase-1 (HO-1) enzyme in cells of mice with sickle cell anemia. In order to do so, they had to insert the rat Hmox-1 gene into the recipient mice. The authors first constructed an albumin promoterdriven Sleeping Beauty transposase with a wild-type rat Hmox-1 transposable element and then inserted both into a single plasmid vector. The idea behind the albumin promoter for the transposase construct was to assure HO-1 expression in the liver. The clones were screened and tested by restriction digest mapping and sequencing followed by protein expression in tissue culture, HO-1 western blot analysis, and HO-1 enzyme activity. The resultant clone expressed wild-type rat HO-1. A nonsense control Hmox-1 vector was then constructed by inserting four basepairs containing an early stop codon into the Hmox-1 sequence of the Sleeping Beauty-wild-type-HO-1 plasmid. The transposon, which harbored the wild-type rat Hmox-1 and the control construct were delivered by hydrodynamic tail vein injections to the mice with sickle cell anemia. Eight weeks after injection, the sickle cell anemia mice had an increase in HO-1 activity and protein expression in liver.

Belcher et al. [8] found a fivefold increase in HO-1 expression of the livers in their sickle cell anemia mice with resultant increased biliverdin and hemin production. Probably as a result, NF- $\mathrm{kB}$ activation and vascular cell adhesion molecule-1 expression was reduced. Belcher et al. [8] speculate that HO-1 gene delivery to the liver is beneficial in sickle cell anemia mice by degrading pro-oxidative heme, releasing anti-inflammatory heme degradation products such as carbon monoxide and biliverdin/bilirubin into the circulation, activating cytoprotective pathways, and inhibiting vascular stasis at sites distal to transgene expression. Those claims may or may not be true. However, what concerns us here is the idea that Sleeping Beauty might have utility as a genetherapy tool, an area currently residing in relative disrepute in the clinical world. However, this sad state-of-affairs could be subject to change and the current report could help.
There are open questions. The somatic gene transfer in this study required only a straightforward intravenous injection of the vector plasmid and its contents. By what mechanisms did the liver cell incorporate this plasmid? No electroporation, no lipofectamine, no viruses, or other tedious methods that plague investigators (including the author) were employed. The ability of Sleeping Beauty to mediate stable, long-term expression in mouse tissues was initially reported by Yant et al. [9], who expressed $\alpha 1$ antitrypsin as a reporter in normal $\mathrm{C} 57 \mathrm{BL} / 6$ mice and human clotting factor IX as a therapeutic gene product in factor-IX-deficient mice. Their studies also relied on injections of plasmid DNA [9]. The applicability of such a therapy is immediately obvious.

The Celebrated Jumping Frog of Calaveras County is an 1867 collection of short stories by Mark Twain. In the story, the narrator tells about the gambler Jim Smiley. Twain describes him as follows: "If he even seen a straddle bug start to go anywheres, he would bet you how long it would take him to get to - to wherever he going to, and if you took him up, he would foller that straddle bug to Mexico but what he would find out where he was bound for and how long he was on the road." The road for Sleeping Beauty is also onerous, but perhaps we should all take a bet that she can jump better than any frog.

Respectfully,

Friedrich C. Luft

\section{References}

1. Voigt K, Izsvák Z, Ivics Z (2008) Targeted gene insertion for molecular medicine. J Mol Med 86:1205-1219

2. Davies DR, Goryshin IY, Reznikoff WS, Rayment I (2000) Threedimensional structure of the Tn5 synaptic complex transposition intermediate. Science 289:77-85

3. Richardson JM, Colloms SD, Finnegan DJ, Walkinshaw MD (2009) Molecular architecture of the Mos1 paired-end complex: the structural basis of DNA transposition in a eukaryote. Cell 138:1096-1108

4. McClintock B (1950) The origin and behavior of mutable loci in maize. Proc Natl Acad Sci U S A 36:344-355

5. Ivics Z, Hackett PB, Plasterk RH, Izsvák (1997) Molecular reconstruction of Sleeping Beauty, a Tc1-like transposon from fish, and its transposition in human cells. Cell 91:501-510

6. Collier LS, Carlson CM, Ravimohan S, Dupuy AJ, Largaespada DA (2005) Cancer gene discovery in solid tumours using transposonbased somatic mutagenesis in the mouse. Nature 436:272-276

7. Ivics Z, Izsvák Z (2006) Transposons for gene therapy! Curr Gene Ther 6:593-607

8. Belcher JD, Vineyard JV, Bruzzone CM, Chen C, Beckman JD, Nguyen J, Steer CJ, Vercellotti GM (2010) Heme oxygenase-1 gene delivery by Sleeping Beauty inhibits vascular stasis in a mural model of sickle cell disease. J Mol Med doi:10.1007/s00109-010-0613-6 (this issue)

9. Yant SR, Meuse L, Chiu W, Ivics Z, Izsvak Z, Kay MA (2000) Somatic integration and long-term transgene expression in normal and haemophilic mice using a DNA transposon system. Nat Genet 25:35-41 